\title{
QUÉ PUEDE DECIRNOS LA \\ RELATIVIDAD GENERAL RESPECTO \\ DE LA FLECHA DEL TIEMPO
}

\author{
Cristian López \\ University of Buenos Aires - Instituto de Filosofia \\ "Alejandro Korn" \\ Departamento de Filosofia \\ Capital Federal \\ Argentina \\ University of Lausanne \\ Switzerland \\ lopez.cristian1987@gmail.com
}

Article info

CDD: 530.01

Received: 14.01.2018; Revised: 13.08.2018; Accepted: 13.08.2018

DOI: http://dx.doi.org/10.1590/0100-6045.2018.V41N3.CL

Keywords:

Arrow of time

General relativity

Temporal invariance

Philosophy of

physics
Palabras claves:

Flecha del tiempo

Relatividad general

Invariancia temporal

Filosofía de la física

\begin{abstract}
Traditionally, from the viewpoint of the philosophy of physics, the problem of the arrow of time has been addressed considering the formal properties exhibited by the fundamental physical laws. In particular, if the laws remain invariant under time reversal, then a privileged temporal direction cannot be distinguished on physical grounds. On the contrary, if a specific law does not remain invariant under time reversal, it is possible to offer a physical and substantial argument for the existence of the arrow of time. However, this strategy has led to several problems, and has contributed to create an atmosphere of skepticism in the field of philosophy of physics, about the potentiality of physics to
\end{abstract}


provide grounds for an asymmetric time. The aim of this article is to show that general relativity seems to be a promising and fruitful field for the philosophical discussion about this matter. It will be argued that it is necessary to put aside the formal properties of the physical laws and the concept of time reversal and, instead, to consider the properties of space-time.

Resumen: Tradicionalmente, desde la filosofía de la física el problema de la flecha del tiempo ha sido abordado en términos de las propiedades que exhiben las leyes físicas fundamentales ante determinadas operaciones, en particular, ante la operación de inversión temporal: si las leyes permanecen invariantes ante inversión temporal, entonces no es posible distinguir un sentido temporal privilegiado desde un punto de vista físico; por el contrario, si una determinada ley no permanece invariante ante inversión temporal, mediante esa ley es posible dar un argumento físico para sostener la existencia de una flecha del tiempo. Sin embargo, esta estrategia ha conducido a numerosos problemas y ha instalado un clima escéptico en el ámbito de la filosofía de la física respecto del potencial de las ciencias físicas para recoger la idea intuitiva de un tiempo asimétrico. En el presente artículo se busca mostrar que la relatividad general constituye un fructífero y prometedor campo de discusión filosófica respecto del problema flecha del tiempo, campo aún no suficientemente explorado. Se argumentará que para abordar esta problemática en el terreno de la relatividad general, es necesario dejar de focalizar en las propiedades formales de las leyes físicas y en el concepto de invariancia temporal, para orientar nuestra búsqueda hacia las propiedades del espacio-tiempo.

\section{INTRODUCCIÓN}

Sin lugar a dudas, una de nuestras creencias más firmes es que el tiempo existe, que el tiempo pasa, que los segundos, minutos y horas son, irremediablemente, devorados por Cronos. La propia tarea de escribir este trabajo muestra el paso temporal: hubo un tiempo, pasado, en el cual la tarea fue emprendida; un tiempo presente, en el 
cual estas líneas son escritas; y un tiempo futuro, donde la tarea estará terminada. Además, las intuiciones temporales del autor están presentes en cada momento de escritura: ¿cuánto tiempo es necesario para terminar este trabajo? ¿Qué fue lo que escribí? ¿Qué escribiré mañana? Los días son ordenados pensando en la próxima página, allí, adelante, todavía imprecisa e inexistente, en algún momento del futuro. La convicción más profunda establece que, en algún sentido, este trabajo ha sido iniciado en el pasado, atrás, hace unos días que recuerdo con precisión; y habría de ser terminado en el futuro, aún incierto, pero allí, adelante.

Sin embargo, todo podría ser industria de un genio maligno, un peculiar genio maligno del tiempo. Posiblemente, el tiempo sea circular y, una vez escrito, el artículo deba volver a escribirse en algún futuro lejano donde el pasado y el futuro se re-encuentran. O, tal vez, nuestras intuiciones temporales están invertidas y el tiempo realmente es como lo consideraban los pueblos aymaras, aquellos caminantes de las alturas de los Andes, para quienes el futuro se hallaba atrás y uno caminaba mirando hacia el pasado, que se encontraba frente a los ojos, como si el mañana fuese el día que está detrás nuestro, como si la tarea de escribir este artículo fuese un caminar de espaldas. Quizás el engaño fuese aún más profundo: quizás dentro del tiempo que transcurre hay otro tiempo, quieto, sin horas ni peso, ni sombra, sin pasado y sin futuro, que nunca vemos. Quizás la tarea de escribir este trabajo nunca existió, quizás estuvo escrito desde siempre, quizás el tiempo fuese una ilusión, la imagen móvil de la eternidad, como decía Platón.

Si bien la naturaleza del tiempo ha sido objeto de inquisición filosófica desde el nacimiento mismo de la filosofía, el problema de la flecha del tiempo como ámbito de discusión y encuentro entre la filosofía y las ciencias físicas es relativamente reciente, remontándose a fines del siglo 
diecinueve y principios del siglo veinte. El origen de la discusión podría situarse en los trabajos de Ludwig Boltzmann, Ernst Zermelo, Johann Josef Loschmidt y Arthur Eddington. En particular, se atribuye a este último haber sido quien acunó la expresión "flecha del tiempo" para referirse al carácter manifiestamente dirigido $y$ asimétrico del tiempo. Naturalmente, desde las primeras reflexiones filosóficas de los helenos respecto de la naturaleza del tiempo hasta las posiciones y discusiones contemporáneas, hubo cambios sustanciales. Permeados por influencias culturales y cosmológicas antiguas, el tiempo cíclico de los filósofos helénicos penduló entre un carácter ilusorio, debido a la inmutabilidad de lo que verdaderamente es (tal como afirmaban los eleáticos), hasta su carácter real, pero dependiente del movimiento de las entidades físicas, que seguían patrones cíclicos (tal como creían los aristotélicos). La discusión filosófica contemporánea, en general, busca fundamentar el carácter asimétrico de un tiempo modernamente lineal que exhibe una sucesión de eventos ordenados según la relación anterior y posterior, donde la física podría tener un papel relevante a la hora de ofrecer un fundamento de por qué el tiempo es como parece ser.

Una de las ortodoxias que se ha instalado y ha imperado en el transcurso de la discusión es la fuerte dependencia entre el fundamento de la flecha del tiempo y la existencia de leyes no invariantes ante inversión temporal: si al invertir el signo de la variable temporal $t$, y de todas las magnitudes definidas en función de ella, se obtiene una evolución físicamente posible, entonces la ley presenta soluciones temporalmente simétricas que reflejan su carácter invariante frente a la operación de "invertir el tiempo". Esto, se alega, no permitiría distinguir la dirección temporal pasado-afuturo de la dirección temporal futuro-a-pasado. Una ley sin esta característica, es decir, una ley que no permanezca invariante ante inversión temporal, es considerada una ley 
que efectivamente permitiría distinguir ambas dirección temporales, pudiendo definir una flecha del tiempo fundamental. Esta fuerte dependencia supuesta en la bibliografía imprimió, quizás involuntariamente, un abordaje preponderantemente local al problema de la flecha del tiempo, frente a un enfoque global que permitiera considerar las propiedades de todo el universo; además condujo a que la atención se centrara en teorías como la termodinámica, la mecánica estadística clásica o la mecánica cuántica.

En el presente artículo se busca mostrar que existe otra vía de investigación donde la relatividad general constituye un fructífero y prometedor campo de discusión filosófica respecto del problema flecha del tiempo, campo aún no suficientemente explorado en el ámbito de la filosofía de la física. Se argumentará que, para abordar esta problemática en el terreno de la relatividad general, es necesario dejar de centrar nuestra atención en las propiedades formales de las leyes físicas y en el concepto de invariancia temporal, para orientar nuestra búsqueda hacia una concepción de la flecha del tiempo en términos de las propiedades topológicas del espacio-tiempo. Este giro en la discusión permitirá comprender el problema de la flecha del tiempo en filosofía de la física desde una perspectiva más amplia.

En vistas a exponer este punto de vista, el presente trabajo se articulará de la siguiente manera: en primer lugar, se reconstruirá y expondrá el problema de la flecha del tiempo desde la perspectiva de la filosofía de la física. En segundo lugar, se presentará la estrategia "ortodoxa" respecto de la flecha del tiempo y las posiciones más representativas respecto de esta problemática, poniendo el énfasis en el concepto de invariancia ante inversión temporal y la relevancia de las propiedades de las leyes físicas para la flecha del tiempo. En tercer lugar, se mostrará cómo la introducción de la relatividad general como terreno de discusión para la flecha del tiempo obliga 
a replantear la problemática en otros términos, atendiendo a otras propiedades físicas, en particular, a las propiedades que presenta el espacio-tiempo. Finalmente, se brindará una reflexión final respecto del potencial filosófico y científico que presenta la relatividad general, proponiendo nuevas reglas de juego en la discusión a la hora de plantear el problema de la flecha del tiempo y de encontrar nuevas respuestas.

\section{EL PROBLEMA DE LA FLECHA DEL TIEMPO DESDE LA FILOSOFÍA DE LA FÍSICA}

El tiempo parece exhibir la propiedad de asimetría: si dos sucesos no son simultáneos, entonces uno es anterior al otro, es decir, existe entre ellos una relación asimétrica. Pero, ¿cuál es la naturaleza de esta asimetría? Además, asumimos que el tiempo posee un carácter unidireccional manifiesto, ya que parece transcurrir hacia el futuro y no hacia el pasado. Cabe preguntarse, entonces, ¿es esta direccionalidad privilegiada una propiedad esencial del tiempo mismo?, ¿es la manera en la que se ordenan naturalmente los sucesos físicos o, simplemente, una apariencia empírica del nivel macroscópico? Las investigaciones en el contexto de la filosofía de la física buscaron encontrar una asimetría material como correlato y fundamento de la asimetría temporal que se nos presenta, permitiendo -al menos en principio- un abordaje del problema de la flecha del tiempo a partir de la estructura nomológica de alguna teoría física. Pero, el formalismo de las teorías físicas actualmente vigentes, ¿permite distinguir una dirección privilegiada del tiempo?

La tradición atribuye a Sir Arthur Eddington (1928: 68) el nombre "flecha del tiempo" para referirse a la naturaleza temporalmente unidireccional de los fenómenos, y es a partir de aquí que el problema de la flecha del tiempo, en 
términos generales, consiste en explicar y dar sentido a este aparente carácter asimétrico y "dirigido" del tiempo y de los eventos naturales.

El problema de la flecha del tiempo admite muchos abordajes y respuestas posibles. Por ejemplo, puede pensarse que el carácter asimétrico y dirigido del tiempo pertenece a nuestra forma subjetiva de percibir los fenómenos. Bajo esta perspectiva, la direccionalidad del tiempo estaría constituida por una flecha psicológica que exigiría una argumentación en términos de cómo la mente humana ordena temporalmente el mundo y determina su evolución a un único sentido posible. También puede considerarse el problema en términos estrictamente metafísicos: la direccionalidad privilegiada del tiempo es una propiedad esencial del tiempo mismo que no admite argumentación en términos a posteriori, sino sólo a priori. También puede establecerse una flecha biológica cuando se pretende coordinar la asimetría temporal con procesos biológicos que exhiben algún tipo de asimetría (v.g., un proceso evolutivo).

No obstante, el abordaje desde la filosofía de la física tiene cierto privilegio epistemológico, y probablemente ontológico, por sobre el resto de los abordajes posibles. Por un lado, el tiempo es una variable fundamental presente en la mayoría de las leyes físicas e, incluso, es objeto de estudio de algunas teorías físicas (v.g., la relatividad general, de la cual hablaremos más adelante). Por otro lado, la discusión en torno al problema de la flecha del tiempo se ha desarrollado, mayoritariamente, en el terreno de la física y la filosofía de la física. La manera de formular el problema en las discusiones filosóficas y científicas al respecto, y también las posibles vías de respuestas, están impregnadas de argumentos provenientes de la física y la filosofía de la física. Por lo tanto, aquí no discutiremos puntos de vista relacionados con temporalidad en otras ciencias o en el ámbito de la experiencia personal, sino que restringiremos 
el problema a los límites de la física. En este contexto, el problema de la flecha del tiempo surge cuando buscamos un correlato físico (en un sentido bastante amplio) a la idea intuitiva de un tiempo con las propiedades de asimetría y unidireccionalidad.

Abordar el problema desde la física y la filosofía de la física nos conduce a prestar atención a las teorías físicas vigentes y atender a qué tipo de relación puede establecerse entre estas teorías y la flecha del tiempo. La estrategia consiste en el intento de encontrar alguna característica material del mundo que pueda ser coordinada de una u otra manera con la direccionalidad temporal (Sklar 1974: 355). Es decir, se intenta reflejar en el formalismo de alguna teoría física vigente la idea de un tiempo asimétrico; para ello, se analizan las propiedades que poseen las diferentes estructuras nomológicas de las teorías y cuál es su comportamiento ante determinadas operaciones formales. Pero, ¿rescatan las leyes de la física estas propiedades del tiempo? Y, en caso de hacerlo, ¿cómo lo hacen?

Supongamos que hay un vaso sobre la mesa. El vaso, en un momento determinado, cae al piso y se rompe en un sinnúmero de pedazos. Quien entra a la habitación y ve lo que ha sucedido puede, rápidamente, reconstruir los sucesos previos: había un vaso sobre la mesa que, luego, cayó al piso y, luego, se rompió. Nunca hemos presenciado la escena temporalmente inversa: un vaso roto en el piso que, espontáneamente, se reconstruye y se ubica en la mesa de manera intacta, con cada molécula de agua en su interior. Llamamos a esta clase de procesos macro-físicos, irreversibles.

Ahora bien, podemos describir esta misma situación desde el punto de vista de la física recurriendo a la estructura formal y conceptual de la mecánica estadística clásica. El vaso con agua es ahora una abrumadora cantidad de partículas que están dispuestas espacialmente de una manera particular, donde cada partícula, en principio, tiene 
una posición y una velocidad determinadas en el instante $t_{0}$ -que corresponde al instante en el que vemos macroscópicamente el vaso sobre la mesa-. En un determinado momento, las partículas dejan de estar confinadas en el espacio definido por el vaso y comienzan a alejarse a distintas velocidades, cambiando radicalmente su disposición espacial: las partículas han cambiado su posición, su velocidad y, ahora, si las tomamos en conjunto, ocupan un espacio completamente diferente, encontrándose ampliamente dispersas. Este instante, $t_{1}$, corresponde a lo que macro-físicamente veíamos como numerosos pedazos de vidrio y agua esparcidos sobre el piso. En principio, podríamos describir la trayectoria de cada partícula según las leyes de la mecánica clásica y determinar una evolución particular del sistema según la cual, en un principio, las partículas se encontraban en un cierto estado y, luego, al cabo de un tiempo, se encuentran en otro estado diferente. Nada hay de extraño allí y el mundo macro-físico encuentra en la descripción del mundo micro-físico una lupa sumamente precisa, acompañada de un formalismo en sintonía con lo observable. Llamemos a esta situación $\boldsymbol{S}_{\mathbf{1}}$.

Sin embargo, todavía considerando el mismo fenómeno pero desde el punto de vista micro-físico, supongamos una situación distinta, a saber, $\boldsymbol{S}_{\mathbf{2}}$ : tomamos el conjunto de partículas que encontrábamos en $t_{1}$, luego de la caída del vaso, invertimos la variable $t, t \rightarrow-t$, e invertimos todas las variables definidas en función de la variable $t$ (v.g., la velocidad o el momento de las partículas). A partir del estado así definido, las partículas comienzan a reagruparse, hasta llegar a ocupar una distribución espacial muy particular: todas ellas conformando el vaso con agua. Claramente quedaríamos perplejos ante esta evolución. Pero tomemos, ahora, una partícula sola entre la 
abrumadora cantidad de partículas en cuestión y hagamos la misma operación. Simplemente, determinaremos la trayectoria de una partícula que primero está en un lugar en un cierto estado y al cabo de un tiempo se encuentra en otro estado y otro lugar. Nada de extraño hay en ello. Pero, entonces, no debería haber nada de extraño si consideramos un número mayor de partículas ni motivos para aplicar un razonamiento distinto si consideramos una partícula o si consideramos una cantidad de $10^{23}$ partículas: las leyes de la mecánica clásica que describen a una partícula describen a todas de la misma manera. Por lo tanto, desde el punto de vista del formalismo físico que describe tanto la situación $\boldsymbol{S}_{\mathbf{1}}$ como la $\boldsymbol{S}_{\mathbf{2}}$, nada hay de sorprendente: $\boldsymbol{S}_{\mathbf{2}}$ es una evolución tan físicamente posible como $\boldsymbol{S}_{\mathbf{1}}$ dado el formalismo de la mecánica estadística clásica. Pero, ¿de qué manera es posible distinguir, en términos físicos, estas dos situaciones evidentemente distintas?

\section{UNA ESTRATEGIA ORTODOXA: T-INVARIANCIA Y FLECHA DEL TIEMPO}

A la luz de este ejemplo particular, precisemos los términos. Llamamos t-invariancia a la propiedad de las leyes físicas de ser invariantes ante inversión temporal, e irreversibilidad a la propiedad que tienen los fenómenos de evolucionar en una dirección temporal y nunca en la contraria. En la bibliografía pueden encontrarse numerosas definiciones de $t$-invariancia (un buen catálogo de ellas puede verse en Savitt 1994, cap. I). Optaré por la siguiente definición, que permite distinguir de manera clara los conceptos de $t$-invariancia y de reversibilidad:

Definición 1: Una ley física $L$ es t-invariante si la ecuación dinámica que la describe es invariante bajo la aplicación del operador de inversión temporal $\mathbf{T}$, el cual lleva a cabo la 
operación $t \rightarrow-t$ e invierte todas las variables dinámicas definidas en función de $t$. Como resultado, si e $(t)$ es una solución de la ecuación dinámica -que describe una evolución posible respecto de $L_{-}, \mathbf{T e}(t)$ es también una solución -también describe una evolución posible respecto de L-. (Castagnino y Lombardi 2009: 3)

El concepto de t-invariancia, como vemos, es una propiedad de las leyes físicas, que depende de la particular forma matemática de las ecuaciones dinámicas que las expresan. Una gran cantidad de leyes físicas parecen tener la propiedad de ser $t$-invariantes: las leyes de la mecánica de Newton, las ecuaciones de campo de Einstein, las leyes de la mecánica cuántica no relativista, las ecuaciones de Maxwell, entre otras. Nótese que las soluciones e $(t)$ y $\mathbf{T e}(t)$ de ecuaciones $t$-invariantes constituyen un par simétrico (en adelante, par de gemelos t-simétricos), donde cada miembro del par es una imagen temporalmente especular de la otra, tal como concebíamos las situaciones $\boldsymbol{S}_{\mathbf{1}}$ y $\boldsymbol{S}_{\mathbf{2}}$.

Definición 2: Una evolución es reversible si no tiene estados de equilibrio finales (o iniciales), es decir, "puntos de no retorno" (Castagnino y Lombardi 2009: 3).

A diferencia del caso anterior, reversibilidad e irreversibilidad son propiedades que se aplican a las evoluciones físicas, representadas por soluciones de las ecuaciones dinámicas, y no a las leyes mismas, representadas por tales ecuaciones. Un ejemplo canónico de evolución irreversible es aquel donde una evolución particular incluye un potencial (o alguna fuerza disipativa), o ciertas características extra-nómicas que dirigen esa evolución hacia una distribución en particular (ver, por ejemplo, Earman 1986 para diferentes sentidos de reversibilidad). Huw Price (1996) establece la siguiente 
analogía para remarcar la diferencia entre ambos conceptos: supóngase una fábrica de sacacorchos que produce sacacorchos que giran de izquierda-a-derecha y sacacorchos que giran de derecha-a-izquierda. Si bien la producción de sacacorchos puede ser simétrica (en el sentido en que se producen iguales cantidades de sacacorchos que giran de izquierda-a-derecha y de derecha-a-izquierda), tal propiedad de la producción no implica que cada saca corcho sea simétrico respecto de su dirección de giro. En efecto, no es el caso: cada saca corcho es asimétrico en su dirección de giro. Las leyes dinámicas "producen" diferentes conjuntos de evoluciones (por ejemplo, evoluciones con $+t \mathrm{y}$ evoluciones con $-t$ ), y pueden ser simétricas (es decir, invariantes) en este sentido. Pero esto no implica que cada evolución sea también simétrica (es decir, reversible): la inclusión de un potencial en la evolución o de condiciones iniciales muy particulares podrían mostrar que la evolución es irreversible, aunque las leyes que la gobiernan sean $t$ invariantes.

En definitiva, las definiciones anteriores ponen de manifiesto que $t$-invariancia y reversibilidad son conceptos distintos que se predican de entidades diferentes. Esta situación no ha sido suficientemente advertida ni subrayada, lo cual ha generado problemas que obstaculizan los debates. Tradicionalmente se ha sostenido que una de las claves para la solución al problema de la flecha del tiempo radica en el concepto de irreversibilidad; pero, al mismo tiempo, se suele afirmar que es necesario encontrar leyes que permitan recoger una direccionalidad del tiempo (es decir, que sean leyes no $t$-invariantes). Aquí hay, al menos, dos confusiones. O bien no hay una clara y precisa distinción entre las propiedades de reversibilidad y $t$ invariancia, y se usan indistintamente sin atender a que corresponden a propiedades de entidades físicas y matemáticas distintas. $\mathrm{O}$ bien se presupone que existe una correlación entre leyes $t$-invariantes y evoluciones 
reversibles y que allí donde hay procesos irreversibles hemos de encontrar leyes no $t$-invariantes que los describan. En ambos casos se pasa por alto que, dadas la Definición 1 y la Definición 2, ambas propiedades admiten, físicamente, todas las combinaciones posibles. El ejemplo de la fábrica de sacacorchos es muy ilustrativo respecto de cómo esto es posible. Además, existen ejemplos más técnicos donde esta distinción permite entender que hay procesos irreversibles descriptos por leyes $t$-invariantes, como leyes no $t$-invariantes cuyas soluciones son reversibles (cf. Castagnino y Lombardi 2005: 75-76).

El enfoque nomológico del problema de la flecha del tiempo en el contexto de la filosofía de la física considera que el correlato físico de la flecha del tiempo consiste en el hallazgo de una ley fundamental no $t$-invariante. Se supone que, si las leyes de la física son $t$-invariantes, deberíamos concluir que la física no recoge en su formalismo una direccionalidad fundamental del tiempo. En otras palabras, la $t$-invariancia de las leyes físicas fundamentales implicaría que la asimetría temporal que percibimos es una mera apariencia sin sustrato físico teórico. Pero, por su parte, si hallamos una ley no $t$-invariante, podríamos generar, de manera no arbitraria, un conjunto de soluciones posibles sólo en una única dirección del tiempo, y no en ambas. Si bien nada en la ley nos señalaría qué dirección es el futuro o el pasado (pues esto es una cuestión puramente nominal), sí nos señalaría una diferencia sustancial entre las dos direcciones, y podríamos convencionalmente bautizar una de ellas como la dirección pasado-a-futuro.

Formulado de un modo más preciso, el argumento de quienes consideran que el hallazgo de una ley no $t$ invariante es relevante para el problema de la flecha del tiempo es muy simple. Podría esquematizarse de la siguiente manera: 
i. Si existen leyes de la física que sean no $t$ invariantes, entonces hay argumentos físicos para establecer la flecha del tiempo.

ii. Existe una ley física que es no $t$-invariante.

iii. Ergo, hay argumentos físicos para establecer la flecha del tiempo.

Nótese que la premisa fundamental es la segunda, y que la primera es el supuesto necesario para formular este argumento. La verdad o no de la segunda premisa es una cuestión que radica, esencialmente, en las propiedades formales de las teorías físicas fundamentales actualmente vigentes. Sin embargo, en este punto es donde surge el problema más acuciante: casi la totalidad de las leyes físicas (al menos las consideradas fundamentales) son $t$ invariantes: las leyes de la mecánica clásica, las ecuaciones de campo de Einstein, la ecuación de Schrödinger, etc.

El tema de la $t$-invariancia o no $t$-invariancia de las leyes de la física ha ocupado una enorme atención en las discusiones sobre la flecha del tiempo. De hecho, la mayoría de los enfoques han hecho depender la solución a esta problemática filosófica del hallazgo de leyes no $t$ invariantes, de tal manera que el problema de la flecha del tiempo desde la filosofía de la física parecer ser el problema de hallar tales tipos de leyes. Para ver con claridad cómo procede esta estrategia argumentativa, expondré a continuación uno de los enfoques que argumenta a favor de una flecha física del tiempo en términos de las propiedades formales de las leyes físicas: el enfoque entrópico (que tuvo una enorme adhesión a mediados del siglo veinte). 


\subsection{UNA FLECHA TERMODINÁMICA DEL TIEMPO.}

Desde su nacimiento en el siglo diecinueve, el problema de la flecha del tiempo ha sido tradicionalmente abordado desde el formalismo y los conceptos de la termodinámica (focalizando en procesos que involucran conceptos como "transferencia de calor", "entropía", "equilibrio y noequilibrio", etc.), constituyendo uno de los enfoques más difundidos en la bibliografía acerca del tema. Es bajo este enfoque que el concepto de entropía y el Segundo Principio de la Termodinámica se han vuelto esenciales. Autores como Ludwig Boltzmann (1895), Hans Reichenbach (1926, 1954), Adolf Grünbaum (1963), David Albert (2000), entre muchos otros, han dado forma a este enfoque que llamaré entrópico. En el libro The Direction of Time (1954), Reichenbach introduce la siguiente definición: "La dirección en la cual la mayoría de los procesos termodinámicos en sistemas aislados ocurren es la dirección positiva del tiempo" (Reichenbach, 1954: 127). En esencia, la idea consiste en que pasado y futuro se definen como los sentidos temporales hacia donde la entropía decrece o aumenta respectivamente, por lo que "si existe una única dirección del tiempo o si la dirección del tiempo alterna, depende de la curva de entropía trazada por el universo" (Reichenbach, 1954: 128).

La tesis del enfoque entrópico, en términos generales, puede expresarse de la siguiente manera:

Tesis general del enfoque entrópico: Pasado y futuro se definen como los sentidos temporales hacia donde la entropía decrece o aumenta respectivamente.

El enfoque entrópico hace depender la asimetría y direccionalidad temporal del Segundo Principio de la Termodinámica, tal como fue formulado anteriormente. Un argumento general para sostener que la dirección temporal pasado-a-futuro es privilegiada en función del Segundo Principio puede reconstruirse del siguiente modo: 
i. La dirección temporal pasado-a-futuro y la dirección temporal futuro-a-pasado son correlacionables y reductibles al gradiente entrópico entre el estado de menor entropía y el estado de equilibrio térmico, y entre el estado de equilibrio térmico y el estado de menor entropía, respectivamente.

ii. La dirección temporal privilegiada es o bien futuro-a-pasado, o bien pasado-a-futuro.

iii. Si la dirección temporal privilegiada es futuro-apasado, entonces el gradiente entrópico de los sistemas debería ir del estado de equilibrio térmico a un estado de menor entropía.

iv. No podrían existir sistemas que presenten tal gradiente (por el Segundo Principio).

v. Por lo tanto, la dirección temporal privilegiada es pasado-a-futuro.

La premisa (i) del argumento presenta lo que llamaré el "supuesto reduccionista" que, intuitivamente, presupone que la dirección temporal privilegiada puede reducirse a una relación no temporal basada en procesos físicos irreversibles. A su vez, si tales procesos están regidos por alguna ley física, justifican nomológicamente la asimetría temporal.

Consideremos un sistema cerrado con dos estados $A$ y $B$, tales que la entropía de $B, S(B)$, es mayor que la entropía de $A, S(A)$. La asimetría física definida a partir del Segundo Principio establece una relación no temporal entre estados donde sólo se permite, nomológicamente, la relación $S(A, B)$ y no la relación $S(B, A)$, donde la relación $S(x, y)$ significa "el estado $x$ tiende al estado $y$ " o "el estado $y$ tiene mayor entropía que el estado $x$ ". Por lo tanto, la asimetría física queda plasmada por la proposición 
$S(A, B) \wedge \neg S(B, A)$. El enfoque entrópico reduce la relación temporal $T(x, y)$, “ $x$ es temporalmente anterior a $y$ ", a la relación no temporal $S(x, y)$ y, por tanto, también privilegia un orden en la relación temporal entre los estados $A$ y $B: T(A, B) \wedge \neg T(B, A)$. Poniendo el problema en términos de $t$-invariancia, al no ser $t$-invariante, el Segundo Principio de la Termodinámica no produce pares $t$ simétricos: si se invierte temporalmente la evolución que conduce a un aumento de la entropía se obtiene una evolución que disminuye la entropía del sistema, lo cual no es una evolución posible de acuerdo a la ley.

Mucha discusión puede rastrearse en la bibliografía respecto de los alcances y problemas del enfoque entrópico. Se exponen a continuación, tres tipos de problemas que pueden ser planteados:

a) Problema conceptual: Se suele entender que la Termodinámica es una teoría fenomenológica, donde las propiedades que describe son propiedades derivadas. Esto se refleja en que, por ejemplo, el concepto de entropía es compatible con diversas configuraciones mecánicas del sistema en cuestión. Esto conduce a pensar si no existe acaso una propiedad más fundamental, no fenomenológica ni derivada, que permita establecer una flecha del tiempo: propiedad que tendrá prioridad conceptual respecto del concepto de entropía (ver Sklar 1974: capítulo V. Para un análisis más general, ver Callender 2016)

b) Problema empirico: Si queremos obtener una flecha del tiempo global, enmarcamos la discusión en el contexto cosmológico. Sin embargo, si adoptamos un enfoque entrópico y buscamos una flecha para todo el universo, presuponemos que es al menos 
posible determinar la entropía para una sección instantánea de él. Pero este supuesto no parece adecuado en el contexto de la cosmología contemporánea: por una parte, no hay una idea clara de cómo definir la entropía producto de campos gravitacionales por lo que sólo se suele considerar la entropía para materia y energía; por otro lado, no todos los espacio-tiempos puede ser divididos en "folios" o "rebanadas" temporales (timelike slices).

c) Problema argumental: El enfoque entrópico cae en cierta circularidad: presupone nuestras intuiciones temporales como, por ejemplo, la relación anteriorposterior. El núcleo del problema es que el argumento termodinámico no debería presuponer que los estados de baja entropía son anteriores a los estados de alta entropía, como así tampoco sostener que ésta aumenta o disminuye: de lo único que da cuenta es de que existe un gradiente entrópico. Este razonamiento forma parte de lo que Huw Price (1996) llama "falacia de doble estándar", y es perfectamente aplicable al caso de los argumentos a favor del enfoque entrópico.

\section{RELATIVIDAD GENERAL Y LA TESIS HERÉTICA DE JOHN EARMAN: EL ESPACIO-TIEMPO Y SUS PROPIEDADES.}

Los numerosos problemas que presentó el enfoque entrópico condujeron a su decadencia hacia la segunda mitad del siglo veinte y a la consecuente opinión generalizada de que, en realidad, la física no era capaz de distinguir la dirección pasado-futuro de la dirección futuropasado, debido al carácter $t$-invariante de sus leyes. Esta 
suerte de pesimismo aún está presente en la filosofía de la física. Tim Maudlin afirma al respecto:

"El tratamiento de esta cuestión es uno de los más peculiares en la literatura filosófica. El enfoque usual configura el problema como sigue: las leyes físicas fundamentales tiene una característica llamada invariancia ante inversión temporal. Si las leyes son invariantes ante inversión temporal, entonces se supone que se sigue que la física misma no reconoce una direccionalidad del tiempo: no distingue, a nivel de ley fundamental, la dirección hacia el futuro de la dirección hacia el pasado." (Maudlin, 2007: 266).

David Wallace presenta el mismo diagnóstico que Maudlin sobre las leyes fundamentales de la física actual:

"En la mayoría de las teorías [físicas] relevantes para nosotros, podemos hallar un operador de inversión temporal que deja invariante la energía de un sistema, la densidad de masa y, esencialmente, toda propiedad de relevancia macroscópica, observable de un sistema." (Wallace, 2012: 265).

Huw Price también expresa este consenso generalizado:

"[el problema] es explicar por qué hay alguna asimetría significativa de las cosas en el tiempo dado que las leyes fundamentales de la física parecen ser (la mayoría) simétricas respecto del tiempo." (Price, 1996: 18).

Como puede apreciarse, en general se acuerda que las leyes fundamentales (como las leyes de la mecánica clásica, las ecuaciones de campo de la relatividad general y las leyes de la mecánica cuántica no-relativista) tienen de hecho la propiedad de $t$-invariancia. Por lo tanto, no serían capaces de distinguir una dirección fundamental del tiempo por sí mismas. Pero, esta escéptica y resignada opinión, ¿ha de extenderse a la totalidad de la física? Como se ha mencionado en la introducción, uno de los objetivos ( $\mathrm{y}$ aspiraciones) de este artículo es mostrar el potencial que tiene la relatividad general como terreno de discusión 
filosófica para el problema de la flecha del tiempo, proponiendo un nuevo tablero donde las piezas deben ser ordenadas de otra manera.

Lo primero a notar es que la discusión se muestra un poco más polifacética que bajo la estrategia usual y los contextos donde se aplicaba (mecánica clásica, termodinámica, mecánica cuántica). En el campo de la relatividad general y la cosmología moderna la discusión se abre a otras opciones, mostrando más aristas. Para poder poner de manifiesto este punto de una manera clara y sencilla, este apartado del artículo se divide en dos partes: en primer lugar, se ofrecerán algunas herramientas conceptuales para poder entender cómo el problema de la flecha del tiempo debe ser replanteado cuando es abordado en el ámbito de la relatividad general; en segundo lugar, se expone una de las tesis que más ha insistido en la necesidad de abandonar la clásica estrategia nomológica respecto de la flecha del tiempo y ha motivado su planteamiento en el corazón de la relatividad general, la Tesis Herética de John Earman.

\subsection{COSMOLOGÍA Y RELATIVIDAD GENERAL.}

La cosmología es la ciencia del universo como un todo. El universo es considerado un objeto físico pasible de ser descripto por leyes físicas, y la interrelación entre el análisis teórico y las observaciones astronómicas ha permitido construir diversos modelos del universo. Existe un consenso bastante generalizado respecto de que (i) la mejor teoría que describe las propiedades del universo a gran escala a partir de leyes físicas es la relatividad general, donde un modelo cosmológico se corresponde con alguna solución a las ecuaciones de campo de Einstein (Hawley y Holcomb 2005: 313), y (ii) el modelo estándar de la cosmología contemporánea está dado por la teoría del Big

Manuscrito - Rev. Int. Fil. Campinas, v. 41, n. 3, pp. 85-123, Jul.-Sep. 2018. 
Bang (Bergstrom 2004: 1). Por lo tanto, la cosmología contemporánea se ocupa de construir modelos cosmológicos que intentan aproximar y describir el universo en el cual vivimos. Estos modelos son soluciones a las ecuaciones de campo de Einstein y añaden diversos parámetros y factores que restringen sus características, a partir de la observación astronómica o de diversas hipótesis físicas, o incluso recayendo en otras teorías o hipótesis teóricas para explicar aspectos particulares del universo y su evolución (como diferentes teorías de gravedad cuántica para explicar los orígenes del universo o las propiedades de agujeros negros, la termodinámica, entre otras).

En esta tarea, se asumen dos principios que desempeñan un papel metodológico en la construcción de modelos cosmológicos:

i. Hay un único universo. Este principio prohíbe, por motivos topológicos, universos desconectados. Como universo y espacio-tiempo son coextensivos y coexistentes, existe un único espacio-tiempo y no existen espacios-tiempos desconectados entre sí. El principio es fundamentalmente metodológico: dado que no podríamos verificar eventos que ocurren en otro universo, metodológicamente asumimos que el universo es sólo uno ${ }^{1}$.

\footnotetext{
${ }_{1}^{1}$ Algunos autores (Brian Green, Stephen Hawking y Sean Carroll, entre otros) han planteado la posibilidad de la existencia de más de un universo (potencialmente, infinitos). La "hipótesis del multiverso", como se la conoce generalmente, ha sido metodológicamente útil en algunas teorías científicas como algunas versiones de las teorías de cuerdas, la teoría de la inflación cósmica e incluso en algunas interpretaciones de la mecánica cuántica. Sin embargo, la hipótesis del multiverso no en absoluto universalmente aceptada por la comunidad de físicos $y$ cosmólogos (por ejemplo, Carlo Rovelli, George Ellis y Paul Davies), ni encuentra asidero en los marcos de la relatividad
} 
ii. Las leyes de la física son las mismas en todo momento y en todo lugar. Si bien no hay necesidad lógica en sostener este principio, no hay evidencia empírica que sustente una consideración contraria al principio. Metodológicamente, se supone esta universalidad para poder operar y razonar en el universo como un todo.

Como se dijo más arriba, los diferentes modelos no sólo deben ajustarse a los marcos teóricos de la relatividad general, sino que no deben contradecir la evidencia empírica acumulada tras décadas de observación astronómica. La cosmología contemporánea sostiene dos hipótesis científicas que son fuertemente sustentadas por la evidencia empírica actual. Ningún modelo cosmológico puede ignorar estas hipótesis $y$, en general, tratan de adecuarse a ellas:

a) Hipótesis cosmológica. El universo es espacialmente homogéneo e isótropo. A gran escala, las características del universo son las mismas en cualquier dirección espacial y en cualquier punto del espacio. La distribución de estrellas, galaxias, nebulosas, etc. (que serían variaciones meramente locales) parecen estar distribuidas de manera "suave" a través del universo: es decir, no hay una concentración mayor de materia en un lugar del universo que en otro, ni en una dirección del universo que en otra, cuando lo consideramos a gran

general y el modelo estándar, sino en otros marcos teóricos y dentro de otros modelos. De todas maneras, el principio de que existe un único universo es un principio metodológico: resulta útil para construir modelos de nuestro universo y establecer cuáles son las propiedades geométricas del espacio-tiempo de nuestro universo. 
escala. Además, el fondo de radiación de microondas es mayormente isótropo. La hipótesis descarta que existan posiciones privilegiadas de observación en el universo.

b) Expansión del universo. Aparentemente, el universo se encuentra en expansión, y las galaxias y cúmulos de galaxias se alejan unas de otras a una velocidad proporcional a sus distancias relativas. Si bien la expansión del universo no es un hecho observable, la inferencia proviene de la observación del corrimiento al rojo que exhiben las galaxias distantes, lo cual es evidencia de que se alejan unas de otras según el efecto Doppler.

Estos principios metodológicos e hipótesis científicas con fuerte apoyo empírico deben ser tomados en cuenta como elementos básicos a la hora de construir un modelo cosmológico con pretensiones de describir nuestro universo. Como se dijo más arriba, un modelo cosmológico -además de adecuarse, en la medida de lo posible, a estos principios- es una solución a las ecuaciones de campo. Naturalmente, estos principios, hipótesis y la relatividad general como teoría marco para explicar las propiedades del universo no son suficientes para obtener una descripción completa y consistente ni del universo como un todo, ni de algunas de sus regiones en particular. La naturaleza y propiedades de los agujeros negros, o el inicio mismo del universo, permanecen inexplicados en esta base teórica. Una explicación científica adecuada de tales objetos y procesos debería involucrar consideraciones cuánticas que involucren la fuerza de gravedad. Hasta el momento, si bien varia teorías han sido propuestos y, en efecto, han resultado ser herramientas fundamentales para entender, o presentar algunas hipótesis sobre, tales fenómenos y la evolución dinámica de nuestro universo (como diferentes versiones 
de gravedad cuántica o teoría de cuerdas), no hay certeza alguna sobre cuál de ellas es la más adecuada. Sin embargo, en este artículo me centraré en los recursos teóricos que la relatividad general (como teoría que sirve de punto de partida para analizar las propiedades geométricas y físicas de nuestro universo) ofrece para fundamentar una flecha del tiempo, por lo que dejaré de lado cualquier consideración que involucre otros marcos teóricos. A continuación, expondré algunos rasgos conceptuales y formales generales de la relatividad general.

\subsection{TEORÍA GENERAL DE LA RELATIVIDAD: ALGUNAS HERRAMIENTAS CONCEPTUALES Y FORMALES.}

Sin lugar a dudas, la teoría de la relatividad ha sido uno de los grandes hitos en la historia de la física y ha alcanzado un grado de divulgación muy amplio. La relatividad especial fue desarrollada por Albert Einstein en 1905, en un artículo llamado "Sobre la electrodinámica de los cuerpos en movimiento". Por su parte, la relatividad general surge diez años más tarde, en la búsqueda de generalizar los resultados de la relatividad especial incluyendo efectos gravitatorios, y se expone principalmente en el trabajo "Los fundamentos de la teoría generalizada de la relatividad" (Einstein 1916).

Einstein presentó la relatividad especial como un resultado de dos principios: el principio de relatividad y el principio de la constancia de la velocidad de la luz en el vacío. Estableciendo una presentación análoga a la relatividad especial, la relatividad general es el resultado de un único principio: el principio de equivalencia. Técnicamente, pueden distinguirse dos principios de equivalencia: un principio débil, que afirma la equivalencia entre masa inercial y masa gravitatoria; y un principio fuerte, que establece que no hay diferencia física entre un cuerpo en caída libre y la ausencia de un campo gravitatorio (para detalles y discusión, ver 
Brown 2005). Otra manera de expresar las dos versiones del principio es comparando la situación física en dos laboratorios, uno en movimiento acelerado y uniforme, y el otro en reposo pero afectado por un campo gravitatorio: el principio de equivalencia débil afirma que los experimentos mecánicos con cuerpos ponderables seguirán el mismo curso en ambos laboratorios; por su parte, el principio de equivalencia fuerte establece que ambos laboratorios son completamente equivalentes y no hay experimento físico por el cual distinguirlos. La equivalencia entre sistemas de referencia en movimiento acelerado y uniforme, y sistemas de referencia en reposo bajo acción de un campo gravitatorio no implica que sistemas de referencia inerciales sean equivalentes a sistemas de referencia no inerciales.

A partir del principio de equivalencia fuerte es posible derivar dos consecuencias de la relatividad general sumamente conocidas: el tiempo "pasa más lento" en presencia de campos gravitacionales, y los rayos de luz se "doblan" si en su camino se interpone un cuerpo que genera un campo gravitatorio. Sin embargo, estrictamente, de acuerdo con la relatividad general esto es sólo una manera de hablar: no existe fuerza gravitatoria sino sólo una influencia de la materia y la energía sobre la geometría del espacio-tiempo. En particular, un cuerpo masivo "curva" la región espacio-temporal circundante haciendo que, por ejemplo, un rayo de luz siga una trayectoria curva en el espacio (pero "recta" en el espacio-tiempo). Esto es lo particularmente novedoso de esta teoría: establece una relación sumamente estrecha entre la geometría del espaciotiempo y la distribución de masa y energía en el universo. Y el vínculo entre estos dos aspectos está dado por las ecuaciones de campo de Einstein.

Geométricamente, el espacio-tiempo de la relatividad general puede no ser "plano" como el espacio-tiempo de Minkovski: la forma geométrica que adquiera el espaciotiempo depende de la distribución de energía y materia en el 
universo. Por lo tanto, en un espacio-tiempo $\left\langle M^{4}, g\right\rangle$ donde $M^{4}$ es una variedad pseudo-Riemanniana cuatridimensional, la métrica $g$ en general no queda representada por un tensor diagonal donde $g_{\mu v}=0$ para $\mu \neq v$; por el contrario, cualquier elemento del tensor métrico puede resultar $g_{\mu v} \neq 0$ De este modo, el tensor métrico representa la curvatura del espacio-tiempo en cada uno de sus puntos. Las ecuaciones de campo de Einstein son las que establecen el vínculo entre la geometría del espaciotiempo y la distribución de masa-energía sobre él:

$$
G_{\mu v}=R_{\mu v}-1 / 2 R g_{\mu v}-\Lambda g_{\mu v}=8 \pi G T_{\mu v}
$$

El lado izquierdo de la ecuación define el tensor de curvatura de Einstein $G_{\mu v}$ en términos de la métrica espaciotemporal $g_{\mu \nu}$, el tensor de Ricci $R_{\mu v}$, el escalar de curvatura $R$ y la constante cosmológica $\Lambda$. El lado derecho de la ecuación representa la distribución de materia y energía en el espacio-tiempo, mediante el tensor de energíamomento $T_{\mu \nu}$. Las ecuaciones de campo dicen, esencialmente, que la forma geométrica que adquiere el espacio-tiempo en una región o en su totalidad está relacionada con la cantidad de materia y energía en esa región o en todo el universo. A su vez, la forma geométrica del espacio-tiempo también determinará la forma geométrica de las trayectorias a través del espacio-tiempo: a mayor cantidad de energía y materia, el tensor de curvatura de Einstein es mayor y la geometría espacio-temporal más "se curva" en esa región, alterando las trayectorias de los cuerpos.

Por último, vale la pena mencionar que cada solución a las ecuaciones de campo de Einstein es un modelo 
cosmológico. Por ejemplo, hay soluciones de "universos vacíos", es decir, sin materia ni energía, donde la geometría espacio-temporal es la del espacio-tiempo de Minkowski. Hay soluciones con universos espacio-temporalmente cerrados sobre sí mismos, ya que la distribución de materia y energía alcanza un índice crítico tal que curva el espaciotiempo hasta cerrarlo sobre sí mismo. Naturalmente, si bien hay infinidad de modelos posibles representando universos posibles, la investigación empírica, la observación astronómica y la contribución de otras teorías físicas selecciona alguno de ellos como candidatos a representar nuestro universo. La teoría actual que busca explicar el origen y la evolución de nuestro universo es la teoría del Big Bang.

Según la teoría del Big Bang, el universo (y el espaciotiempo) comenzó a partir de condiciones iniciales sumamente especiales: una singularidad sumamente densa, increíblemente pequeña y muy caliente a partir de la cual surgió un universo en expansión (para más detalles, if. Bergstrom y Goobar 2004, Capítulos 10-12, Hawley y Holcomb 2005, Parte IV). Esta situación queda representada por los modelos de Friedmann-LamaitreRobertson-Walker (FLRW, en adelante), que son una solución a las ecuaciones de campo de Einstein mediante la métrica de Robertson-Walker. Según los modelos FLRW, el universo es isótropo, homogéneo y se encuentra en expansión.

\subsection{LA HEREJía DE LA FLECHA DEL TIEMPO DE JOHN EARMAN}

La Herejía de la Flecha del Tiempo de John Earman (1974) pretende ser una bisagra en el problema de la flecha del tiempo, problema que, como el propio autor diagnostica, involucra muchas confusiones conceptuales. El espíritu de la Herejía de Earman consiste en desechar por 
completo la estrategia argumentativa ortodoxa que se expuso anteriormente. Según la nueva perspectiva, podría pensarse que el problema de la flecha del tiempo no depende de la no $t$-invariancia de las leyes físicas. Más radicalmente: la $t$-invariancia o no $t$-invariancia de las leyes físicas no nos dice nada acerca de la dirección del tiempo. La Herejía de la Flecha del Tiempo sigue esta línea argumentativa, redirigiendo la discusión al terreno relativista como el terreno "natural" de indagación sobre las propiedades del tiempo; pero la discusión no se enfoca en el estudio de las propiedades formales de las ecuaciones de la teoría, sino en la consideración de las propiedades intrínsecas del espacio-tiempo. La Herejía puede formularse en los siguientes términos (Earman 1974: 20):

Herejia de la Flecha del tiempo: (i) Si existe una flecha del tiempo, ésta es una característica intrínseca del espacio-tiempo (expresable en términos de orientación temporal), que no necesita y no puede reducirse a características no temporales. (ii) $\mathrm{La}$ existencia de una flecha del tiempo expresable en términos de orientación temporal no depende, crucialmente, de la irreversibilidad, como los reduccionistas suponen.

El primer punto de la tesis herética introduce un concepto nuevo que no ha aparecido en la discusión hasta el momento: el concepto de orientación temporal. Resolver el problema de la flecha del tiempo no consiste, si asumimos la tesis herética, en hallar leyes no $t$-invariantes, sino en decidir si es posible definir una orientación temporal del espacio-tiempo. Esta orientación temporal no es una propiedad derivada a partir de otras asimetrías físicas ni espacio-temporales, sino una asimetría evidenciada en la estructura misma del espacio-tiempo. El segundo punto desvincula el problema de la flecha del tiempo del concepto 
de irreversibilidad. Puesto que Earman no distingue -como se ha hecho en el presente trabajo- entre $t$-invariancia y reversibilidad, sino que los trata como conceptos coextensivos, su tesis puede traducirse como afirmando que se ha sobreestimado el papel de los conceptos de $t$ invariancia y de reversibilidad para solucionar el problema de la flecha del tiempo.

La Herejía de Earman busca desarticular el enfoque que ha privilegiado el papel de la irreversibilidad y la no $t$ invariancia, asumiendo una postura no reduccionista y global. Los enfoques como el entrópico, que apelan a la ruptura de la simetría temporal mediante leyes físicas no $t$-invariantes, vinculan de manera directa la asimetría temporal con alguna asimetría en la evolución de una magnitud física, asimetría descripta por una ley no $t$-invariante. En su lugar, Earman propone que la discusión sea orientada al campo de la relatividad general y de las propiedades que el espaciotiempo exhibe: si existe una asimetría temporal, ésta debe quedar evidenciada como una propiedad del propio espacio-tiempo.

\section{Pateando el tablero: Relatividad General $Y$ FLECHA DEL TIEMPO.}

La tesis de Earman establece un gozne en la discusión, ya que insta a abandonar los presupuestos básicos sobre los cuales ha sido construida la discusión filosófica respecto de cómo fundamentar físicamente nuestras intuiciones temporales. Y esto es así porque plantear el terreno de la discusión en el ámbito de la relatividad general no es un mero cambio de ambiente, no es solamente un nuevo terreno en el cual aplicar nuestras usuales herramientas formales y conceptuales. Si uno desea abordar el problema de la flecha del tiempo en el ámbito de la relatividad general, guiado por el espíritu herético de Earman, 
entonces debe abandonar tales herramientas, abandonar los conceptos previos y visualizar el problema (y las soluciones) desde un nuevo punto de vista relativista.

Por ejemplo, podría pensarse que existe un problema muy elemental a la hora de discutir filosóficamente la naturaleza del tiempo y su direccionalidad en el campo de la relatividad general: las ecuaciones fundamentales de la teoría, las ecuaciones de campo, son $t$-invariantes, es decir, si $h_{i j}=h_{i j}\left(t, x^{1}, x^{2}, x^{3}\right)$ es una solución de las ecuaciones, entonces $h_{i j}=h_{i j}\left(-t, x^{1}, x^{2}, x^{3}\right)$ es también una solución (cf. Halliwell 1994), donde $h_{i j}$ es la métrica Riemmanian positivamente definida. Esto parece problemático: si la ley fundamental de la teoría es $t$-invariante, nada podría decirnos la relatividad general acerca de por qué percibimos el tiempo como asimétrico. Al parecer, se continúa propiciando la escéptica y resignada conclusión de que el tiempo que percibimos es una ilusión humana, demasiado humana.

En este punto es donde la tesis herética de Earman nos advierte que hemos prestado demasiada importancia a las propiedades formales de las leyes físicas. Pero hay un sentido más profundo aún para desconfiar de la estrategia tradicional, en particular cuando estamos tratando con objetos como el espacio-tiempo o el universo: el concepto de $t$-invariancia a escala global no tiene sentido. Cuando utilizamos el operador de inversión temporal para representar una evolución (una solución) que es físicamente posible que ocurre en una dirección o en otra, es físicamente claro lo que queremos decir: primero la imaginamos en un tiempo positivo, con su velocidad y momento en positivo, y luego imaginamos la evolución desandando sus pasos, en un tiempo negativo, con las magnitudes definidas en función del tiempo también invertidas. Pero cuando queremos hablar del espacio- 
tiempo o el universo como un todo, ¿qué sentido físico tiene la inversión temporal, como si hubiese un punto externo (un tiempo externo) desde el cual pudiera decirse que el tiempo está siendo invertido? Esto se expresa más claramente cuando vemos un problema similar pero en términos espaciales: ¿qué sentido físico tiene mover el universo 3 metros a la izquierda o rotarlo $180^{\circ}$ ? El tiempo no es algo sustancialmente distinto al espacio, sino una dimensión de una totalidad espacio-temporal; por lo tanto, la analogía es totalmente válida para mostrar que preguntarnos por cómo luciría un universo con un tiempo corriendo en la dirección futuro-pasado, carece de sentido físico. Por lo tanto, el presupuesto conceptual básico de plantear el problema de la flecha del tiempo en términos de $t$-invariancia no es aplicable al caso de la relatividad general. Con lo cual, el carácter $t$-invariante de las ecuaciones de campo es, meramente, una anécdota formal que no constituye un argumento sustancial contra la posibilidad de fundamentar la idea de un tiempo asimétrico a partir de la relatividad general.

Pero, entonces, ¿hacia qué propiedades debemos dirigir nuestra atención? Naturalmente, hay que observar las soluciones a las ecuaciones de campo de Einstein. El problema es que las ecuaciones tienen muchísimas soluciones posibles (de hecho, infinitas) y, obviamente, no todas representan nuestro universo. Por ejemplo, existen soluciones a las ecuaciones de campo donde los modelos cosmológicos no permiten fundamentar una flecha del tiempo. Por ejemplo, el famoso lógico y matemático Kurt Gödel propuso en 1949 un modelo cosmológico que contiene curvas temporales cerradas, es decir, un modelo cuyo espacio-tiempo presenta regiones donde la variedad se curva sobre sí misma conteniendo "caminos temporales cerrados" posibles para los objetos físicos. Esta idea ya era conocida por Einstein y permitía paradojas temporales como viajes al pasado o violaciones a la asimetría causal. Es 
decir, que si una partícula viaja a través de una curva temporal cerrada, volverá, al cabo de un tiempo, al mismo punto del que partió. De forma general, tal como Gödel presenta a su universo, puede darse la siguiente situación. Consideremos una partícula que viaja a través del espaciotiempo y señalemos dos eventos sobre su línea de mundo, digamos $p$ y $q$. Supongamos que $p$ antecede temporalmente a $q$ en la línea de mundo de la partícula, es decir, sobre dicha línea de mundo primero ocurre $p$ y luego ocurre $q$. Si la partícula viaja a través de una curva temporalmente cerrada que vincula a los eventos $p$ y $q$, habrá un sentido en el cual $q$ antecede temporalmente a $p$.

Sin embargo, el modelo de Gödel es sólo una de las soluciones posibles a las ecuaciones de campo de Einstein. Solución que, en nuestros días, no tiene respaldo empírico y por tal motivo es descartada como modelo cosmológico. Algunos autores han ido más allá de la mera búsqueda de modelos cosmológicos que sean soluciones a las ecuaciones de campo y han argumentado que la asimetría temporal en el universo proviene de la asimetría entre las condiciones iniciales y finales del universo. Por ejemplo, a diferencia del modelo de Gödel, el modelo de un universo en bloque que evoluciona (UBE), propuesto por George Ellis, considera la existencia de una evolución temporal a escala cosmológica a partir de las particularísimas condiciones iniciales que dieron comienzo al tiempo mismo. La propuesta de Ellis se enmarca en un enfoque más general que considera la existencia de una jerarquía compleja de niveles, en los cuales existen flechas del tiempo locales: una flecha del tiempo en el nivel cuántico, una en el nivel termodinámico, una en los organismos vivos, etc. Estas estructuras están relacionadas entre sí, permitiendo recorrerlas en dirección abajo-hacia-arriba [bottom-up effects] y en dirección arribahacia-abajo [top-down effects], generando efectos en los niveles vecinos. Esta última dirección es la más relevante ya que simula un "efecto cascada", donde los niveles más 
complejos se realizan de manera múltiple en los niveles inferiores (Ellis 2013: 244). Según Ellis, en un modelo como UBE, el pasado es, evidentemente, diferente del futuro, lo cual se representa por un espacio-tiempo que crece con el tiempo: el pasado permanece fijo e inalterable y el futuro todavía permanece abierto. Pasado y futuro son separados por el presente, donde el pasaje objetivo del tiempo se evidencia en una continua progresión donde lo indeterminado se vuelve determinado. No sólo las cosas que suceden en el espacio-tiempo se determinan, sino la propia estructura espacio-temporal se va determinando a medida que las cosas sobre ella se determinan (Ellis 2013: 258). De este modo, el universo que nos propone Ellis pretende recoger, en su propia estructura, un pasaje de tiempo objetivo y físicamente real, dado por la Flecha Maestra del Tiempo.

El problema con el enfoque de Ellis es que sólo toma en cuenta las condiciones iniciales del universo, sin interrogarse si acaso éstas no pueden ser simétricas en relación a las condiciones finales. Es claro que el núcleo del argumento de Ellis radica en que no existe tiempo antes del inicio del universo y que la expansión de éste señala la dirección hacia la cual el parámetro $t$ aumenta. Sin embargo, no parece que Ellis esté diciendo nada nuevo ni sustancial para el problema de la dirección temporal. En su lugar, parece presuponer que es absolutamente obvio que el inicio del universo es el inicio del tiempo y que podemos señalar, con total claridad, cuál es, determinando así una dirección. Pero al no considerar las condiciones finales del universo, si son o no físicamente simétricas con respecto a las condiciones iniciales, el argumento, creo, resulta incompleto e, incluso, parece cometer una petición de principio: se busca fundamentar cuál es la dirección pasadoa-futuro pero se la presupone al considerar como condición inicial el inicio del universo junto con el inicio del tiempo. En el fondo, todo parece un juego verbal al hacer confluir 
en un evento físico (el Big Bang) diferentes "inicios" (del universo, del tiempo, etc.) sin considerar las condiciones finales: si el Big Bang y el Big Crunch son físicamente simétricos, no habría razones sustanciales para decir que el Big Bang es el inicio y el Big Crunch es el fin del universo; y, con ello, no habría razones para hablar de un inicio del tiempo en el Big Bang.

Sin embargo, estas críticas no deben impulsarnos a renunciar a continuar la investigación filosófica en el marco de la relatividad general. Existen otras propiedades del universo que pueden ser analizadas en busca de la asimetría temporal.

El universo, tal como es considerado por la cosmología contemporánea, es un objeto espacio-temporal de cuatro dimensiones. Como indicamos más arriba, es un objeto que puede ser representado geométricamente en términos de una variedad cuatri-dimensional pseudo-Riemanniana, cuyas propiedades están vinculadas con la distribución de materia y energía a través de las ecuaciones de campo de Einstein. El universo o el espacio-tiempo, como objeto, tiene la propiedad de ser asimétrico o simétrico a lo largo de la dimensión temporal. Si pensamos en una flecha como un objeto geométrico, decimos que es un objeto asimétrico en una de las direcciones espaciales. $\mathrm{Si}$ dividimos transversalmente la flecha en dos mitades, vemos que las partes resultantes no son iguales. En algún sentido, en una flecha como objeto geométrico podemos distinguir dos direcciones: la dirección punta-a-cola y la dirección cola-apunta, ambas sustancialmente distintas en virtud de la propia geometría del objeto. El razonamiento de fondo para argumentar en favor de una asimetría temporal global del espacio-tiempo es análogo: para fundamentar la flecha del tiempo es necesario que se puedan distinguir las dos direcciones temporales apelando a una propiedad que sea intrínseca a la variedad, como lo es su propia geometría. 
Sobre esta base, la cuestión de si es posible definir una flecha del tiempo global puede formularse en términos de si el universo, o el espacio-tiempo como objeto geométrico, tiene la propiedad de ser asimétrico o no a lo largo de la dirección temporal. ¿De qué manera, en principio, podemos determinar la asimetría temporal del universo? Dicha asimetría debería evidenciarse en una distribución asimétrica de la materia y la energía a lo largo de la dimensión temporal: la asimetría en la distribución de materia y energía se manifiesta también como una asimetría geométrica del espacio-tiempo mismo. Un enfoque que parta de estas premisas se fundamenta en considerar que son las propiedades geométricas del espacio-tiempo las que tienen prioridad conceptual respecto de cualquier otra propiedad física: es necesario que el espacio-tiempo posea ciertas características geométricas para que sea posible definir ciertas magnitudes físicas o determinar ciertas propiedades para todo el universo de manera asimétrica. Esta forma de razonar es la que conduce a la formulación de un enfoque global geométrico, que ha sido propuesto en una serie de trabajos, principalmente por Mario Castagnino y Olimpia Lombardi (2003, 2004, 2005, 2009): argumentando contra la posibilidad de abordar el problema de flecha del tiempo desde una perspectiva local, los autores consideran que sólo desde una perspectiva global, que actúa como telón de fondo para todas las asimetrías locales, es posible definir una direccionalidad temporal. $\mathrm{Y}$, si el enfoque es global, la relatividad general es el marco teórico adecuado para la discusión. ¿Cuál es la estrategia de un enfoque que atienda a la geometría del universo, entonces? Hallar alguna asimetría geométrica en la estructura del espacio-tiempo a lo largo de la dimensión temporal a partir de la relatividad general. 


\section{CONCLUSIONES}

A poco más de 110 años de la formulación de la relatividad general, el potencial teórico y los alcances filosóficos de la teoría no han sido todavía totalmente recorridos ni mucho menos agotados. El vínculo interdisciplinario entre las ciencias físicas y la filosofía permite a la investigación alcanzar nuevos límites y traspasarlos, y mostrar que allí, donde aparentemente no había nada más que decir, puede esconderse un mundo: viejos problemas filosóficos pueden ser iluminados desde diversas teorías científicas, así como diversas teorías científicas pueden ser enriquecidas por la mirada y la inquisición filosófica. Este artículo pretendió mostrar exactamente eso. El problema de la naturaleza del tiempo es un antiguo y clásico problema metafísico de la filosofía, y prácticamente de cualquier cultura. Como tema de investigación científica y filosófica, el problema de la flecha del tiempo es de reciente nacimiento y, sin lugar a dudas, ha mostrado un extenso campo de reflexión conceptual. Campo en el cual la relatividad general ha tenido una incidencia escasa en términos comparativos. Recién hacía el último cuarto del siglo veinte se ha comenzado a insinuar su vital importancia y el potencial de sus posibles aportes. El trabajo buscó exponer de manera sistemática el problema de la flecha del tiempo, desde una perspectiva crítica y no lineal, y mostrar cómo la relatividad general ha incidido en la discusión. Además, intentó argumentar en favor de un giro más manifiesto de la discusión hacia su terreno, exponiendo cómo debe reconstruirse la discusión en este marco relativista y cuáles son sus posibles vías de investigación. Este camino recién está empezando a ser recorrido, y ojalá los 200 años de la relatividad general sean conmemorados mostrando los frutos de la investigación interdisciplinaria en este ámbito. 


\section{BIBLIOGRAFÍA}

AlberT, D. Time and Chance. Cambridge, MA: Harvard University Press, 2000.

Bergström, L. Y GoOBAR, A. Cosmology and Particle Astrophysics. Dordrecht: Springer and Praxis Publishing, 2004.

BOLTZMANN, L. "On certain questions of the theory of gases", en Nature, 51: 413-415, 1895.

Brown, H. Physical Relativity. Oxford: Oxford University Press, 2005.

CALLENDER, C. "Thermodynanic asymmetry in time", en Edward N. Zalta, The Stanford Encyclopedia of Philosophy, URL

=https://plato.stanford.edu/archives/win2016/ entries/time-thermo/, 2016.

CASTAgninO, M. y LOMBARDI, O. "Time asymmetry as universe asymmetry", pp. 11-15, en O. Descalzi, J. Martínez y S. Rica (eds.), Instabilities and nonequilibrium Structures IX, (Dordrecht: Kluwer), 2004.

, LARA, L. y LOMBARDI, O. “The cosmological origin of time asymmetry", en Classical and Quantum Gravity, 20: 369-391, 2003. , LOMBARDI, O., y LARA, L. "A global and nonentropic approach to the problem of the arrow of time”, pp. 74-108, en A. Reimer (ed.), Spacetime Physics Research Trends. Horizons in World Physics. New York: Nova Science, 2005.

y LOMBARDI, O. "The global non-entropic arrow of time: from global geometrical asymmetry to local energy flow", en Synthese, 169: 1-25, 2009. 
EARMAN, J. "An attempt to add a little direction to "The Problem of the Direction of Time", en Philosopby of Science, 41: 15-47, 1974.

EdDington, A. The Nature of the Physical World. Cambridge: Cambridge University Press, 1928.

ELLIS, G. "The arrow of time and the nature of spacetime", en Studies in History and Philosophy of Modern Physics, 44: 242-262, 2013.

GÖDEL, K. "An example of a new type of cosmological solution of Einstein's field equations of gravitation", Review of Modern Physics, 21: 447-450, 1949.

Grünbaum, A. Philosophical Problems of Space and Time. New York: Alfred A. Knopf, 1963.

HALLIWELL, J. "Quantum cosmology and time asymmetry", pp. 369-389. en J. Halliwell, J. PerezMercader y W. Zurek (eds.), Physical Origins of Time Asymmetry. Cambridge: University Press, 1994.

Hawley, J. Y HolCOMB, K. Foundations of Modern Cosmology. Oxford: Oxford University Press, 2005.

Maudlin, T. Metaphysics Within Physics. New York: Oxford University Press, 2007.

PRICE, H. Time's Arrow and Archimedes' point: New Directions for the Physics of Time. New York: Oxford University Press, 1996.

ReICHenbach, H. The Philosophy of Space and Time. New York: Dover Publications, 1928.

The Direction of Time. Berkeley: University of California Press, 1956.

SAVITT, S. "The replacement of time", en Australasian Journal of Philosophy, 72: 463-474, 1994. 
SKLAR, L. Space, Time and Spacetime. Berkeley: University of California Press, 1974.

WALLACE, D. "The arrow of time in physics", pp. 262-281, en Adrian Bardon and Heather Dyke (Eds.), A Companion to the Philosophy of Time, Wiley-Blackwell, 2012.

(cc) $\mathbf{E Y}$

Manuscrito - Rev. Int. Fil. Campinas, v. 41, n. 3, pp. 85-123, Jul.-Sep. 2018. 